affirming the key role of IL-1 and CMF in postinfarction cardiac remodelling.

Funding Funded by a British Heart Foundation project grant.

\section{BAS/ EFFECT OF P38- $\alpha$ GENE SILENCING ON CYTOKINE AND BSCR42 MATRIX METALLOPROTEINASE EXPRESSION BY HUMAN CARDIAC MYOFIBROBLASTS}

\author{
doi:10.1136/hrt.2010.205781.53
}

J K Sinfield, A Das, K E Porter, N A Turner. Division of Cardiovascular and Neuronal Remodelling, Multidisciplinary Cardiovascular Research Centre (MCRC), University of Leeds, Leeds, UK

Cardiac myofibroblasts (CMF) have a key role in the remodelling of the heart that occurs following a myocardial infarction. This remodelling can be initiated by increased myocardial levels of proinflammatory cytokines (eg, interleukin (IL)-1), that can stimulate cardiac myofibroblasts to express other proinflammatory cytokines and matrix metalloproteinases (MMPs). The p38 mitogenactivated protein kinase (MAPK) signalling pathway is also known to be detrimental in the myocardial remodelling process. There are four known p38 MAPK subtypes $(\alpha, \beta, \gamma$ and $\delta$ ) and CMF express the $\alpha, \gamma$ and $\delta$ subtypes. The aim of this study was to determine the role of individual p38 subtypes in mediating IL-1-induced increases in proinflammatory cytokine and MMP expression in cultured cardiac myofibroblasts from different patients. Pharmacological inhibitors of $\mathrm{p38}-\alpha / \beta$ (SB203580) and $p 38-\alpha / \beta / \gamma / \delta$ (BIRB-0796) inhibited IL-1induced IL-6 and MMP-3 mRNA expression to similar extents, suggesting a key role for p38- $\alpha$. Neither inhibitor affected IL-1induced IL-1 $\beta$ or MMP-9 mRNA levels. Gene silencing with p38- $\alpha$ siRNA oligonucleotides selectively reduced $\mathrm{p} 38-\alpha$ protein expression by $>95 \%$ and prevented consequent phosphorylation of the downstream substrate MAPKAPK2. However, p38- $\alpha$ silencing did not markedly inhibit phosphorylation of the MAPKAPK2 substrate HSP27. Furthermore, p38- $\alpha$ gene silencing did not reduce IL-1-induced expression of IL-6 or MMP-3 (or IL-1 $\beta$ or MMP-9). Thus, in contrast to results with pharmacological p38 MAPK inhibitors, gene silencing of p38- $\alpha$ in human cardiac myofibroblasts did not inhibit IL-1-induced IL-6 and MMP-3 expression. This raises interesting questions about pharmacological versus molecular strategies for inhibiting p38 MAPK subtypes in the remodelling heart.

Funding Funded by a British Heart Foundation project grant.

\section{BAS/ X-BOX BINDING PROTEIN 1 SPLICING IS CRUCIAL IN BSCR43 ENDOTHELIAL CELL PROLIFERATION}

doi:10.1136/hrt.2010.205781.54

Lingfang Zeng, Qingzhong Xiao, Hongling Li, Andriana Margariti, Yanhua Hu, Qingbo Xu. Cardiovascular Division, King's College London BHF Centre, London, UK

The X-box binding protein 1 (XBP1) is an endoplasmic reticulum stress response transcription factor. Our previous study showed that sustained activation of XBP1 splicing led to atherosclerosis development (Zeng et al. PNAS 2009). However, the function of XBP1 expression and splicing in endothelial cells and angiogenesis remains unclear. To study this issue, we generated XBP1 knockout mice by deletion of exons 1 and 2 of the gene. XBP1-null (XBP1-/-) embryos at E12.5 showed growth retardation and pale colouration phenotype. The average body weight of XBP1-/-embryos was $40 \%$ less than that of wild-type $(\mathrm{XBP} 1+/+)$ animals. The blood vessel density in XBP1-/- embryos was significantly reduced, owing to a smaller number of CD31+ and Flk1+ cells. In in vitro culture of whole embryonic cells, XBP1-/- cells grew significantly more slowly and lost response to VEGF stimulation. To study the mechanism of XBP1-mediated cell growth, human umbilical vein endothelial cells were treated with VEGF that transiently activated IRE1 $\alpha$ phosphorylation at Ser724. The mRNA movement inhibitor, cycloheximide, ablated VEGF-induced IRE1 $\alpha$ phosphorylation and XBP1 splicing. Co-immunoprecipitation assay showed that there was interaction among KDR, IRE1 $\alpha$ and unspliced XBP1 (XBP1u), which could be increased by VEGF treatment. Further experiments demonstrated that the C-terminal region of $\mathrm{KDR}$ and the kinase domain of IRE1 $\alpha$ are responsible for their interactions. MTT and $\mathrm{BrdU}$ incorporation assays indicate that transient activation of XBP1 splicing increased while long-term activation decreased endothelial survival and proliferation. Knockdown of XBP1 or IRE1 $\alpha$ ablated VEGF-induced proliferation in endothelial cells. Immunofluorescent staining and TOP Flash reporter assay showed that overexpression of XBP1s increased $\beta$-catenin translocation into the nucleus. Thus, this study demonstrated for the first time that XBP1 is crucial for endothelial growth and angiogenesis, in which VEGF-stimulated IRE1 $\alpha / X B P 1$ splicing system and interaction with $\beta$-catenin are key elements, indicating a potential target of XBP1 for protecting endothelial integrity.

\section{BAS/ AKT ISOFORMS IN ISCHAEMIC PRECONDITIONING BSCR44}

doi:10.1136/hrt.2010.205781.55

S P Kunuthur, D J Hausenloy, D M Yellon. The Hatter Cardiovascular Institute, University College London, UK

Akt/protein kinase $\mathrm{B}$ is a key mediator of protection against myocardial ischaemia-reperfusion (I-R) injury. Previous studies have shown that Akt is activated in response to cardioprotective ischaemic preconditioning (IPC) stimulus. However, there are three different Akt isoforms, of which Akt1 and Akt2 are the most abundant in the heart, and it is unclear which isoform is crucial for IPC-induced cardioprotection. The aim of this study was to identify the Akt isoform which is essential for mediating IPC-induced cardioprotection.

Mice deficient in Akt1 or Akt2 were subjected to in vivo regional myocardial ischaemia for $30 \mathrm{~min}$ followed by reperfusion for $2 \mathrm{~h}$ with or without a prior IPC stimulus, comprising $5 \mathrm{~min}$ of ischaemia followed by 5 min of reperfusion. Infarct size and area at risk were determined by differential staining with tetrazolium chloride and Evans Blue dye. Mice lacking either single or both alleles for Akt1 were not amenable to IPC (see table 1). In contrast, only mice lacking both alleles for Akt2 were resistant to IPC, whereas mice heterozygous for Akt2 were still amenable to IPC (see table 1).

Table 1 Infarct sizes expressed as a percentage of the area at risk

\begin{tabular}{llll}
\hline & $+/+$ & $+/-$ & $-/-$ \\
\hline Akt1 & & & \\
I-R & $45.5 \% \pm 2.6 \%$ & $45.3 \% \pm 5.1 \%$ & $47.2 \% \pm 7.2 \%$ \\
I-R+IPC & $28.9 \% \pm 1.4 \%{ }^{*}$ & $40.5 \% \pm 8.0 \%$ & $37.9 \% \pm 4.0 \%$ \\
Akt2 & & & \\
I-R & $41.8 \% \pm 4.0 \%$ & $46.2 \% \pm 4.7 \%$ & $46.4 \% \pm 5.6 \%$ \\
I-R +IPC & $30.2 \% \pm 2.3 \%{ }^{1}$ & $20.7 \% \pm 2.0 \%{ }^{1}$ & $35.9 \% \pm 5.0 \%$ \\
\hline
\end{tabular}

${ }^{*} \mathrm{p}<0.05$ versus respective $\mathrm{I}-\mathrm{R}$ group.

In conclusion, it appears that Akt1 but not Akt2 is essential for cardioprotection conferred by ischaemic preconditioning. 